\title{
Assessment of new induction program on the level of patient anxiety during third molar surgery: A randomized trial
}

Randa Alfotawi*, Abdulrahuman AlHowikan, Alia Alfadhel, Sangeetha Premnath, Jamilah Tawahri, Anfal Alhamid and Shaima Bahammam

Oral and Maxillofacial Department, Dental Faculty, King Saud University, Riyadh, Saudi Arabia

\begin{abstract}
The authors hypothesized that an audio-visual presentation providing information regarding the removal of an impacted mandibular third molar could improve patient knowledge of the surgical procedure and its possible complications. This possibly could decrease anxiety during the surgery. This randomized clinical trial included young adult patients who required surgical removal of an impacted mandibular third molar and fulfilled the predetermined criteria. Forty-two patients met the inclusion criteria; two patients refused to look at the audio-visual presentation. For both groups, heart rate (HR) was recorded beat-by-beat using an HR sensor (POLAR H1, UK) connected to an ActiGraph wGT3X-BT, USA. The Modified Dental Analogue Scale was used to subjectively record the anxiety during the surgery. Those who watched the audio-visual presentation before surgery had lower HR reading compared with those who received verbal instructions. These differences in mean HR reading were statistically significant for the following surgical stages: drilling, suturing, and leaving the clinic. The audio-visual informed group had lower self-reported anxiety scores than did the verbally informed group. These results suggested that providing an audio-visual presentation about the surgical procedures in our routine clinical practice could aid in alleviating anxiety and thereby reduce surgical complications.
\end{abstract}

\section{Introduction}

The essential nature of a patient-doctor relationship can be emphasized through patients' enlightenment on the necessary steps to be taken during an oral surgery. This enlightenment is done, prior to the actual procedure. As a way of addressing certain discrepancies and malpractices observed on the part of medical professionals through the years, it became necessary to keep patients informed of the entire process prior to medical procedures, as well as obtain patients consent, which in totality, amount to involving the patients in their own medical care [1]. Also, instances and documentary evidence point to the fact that patients' anxiety may be reduced after being dealt with thorough preoperative information [1]. Furthermore, it has been shown that anxiety is related to the perception and tolerance of pain. Therefore, a patient's anxiety may impair how well the practitioner performs delicate and complex treatment procedures. It is well known that dentists consider treatment to be technically superior when patients experience less stress [2].

Anxiety can increase surgical risk to patients, particularly in those with undiagnosed conditions for example subclinical heart ischaemia [3,4]. Clinically, during simple dental procedures, such as low complexity restorative treatment, anticipating pain can increase anxiety and create a stressful situation with possible cardiovascular alterations [5]. Monitoring heart rate beat-by-beat, which is regulated by the sympathetic and parasympathetic nervous system, is a noninvasive method that is easy to perform in clinical practice [6-8].

Nonetheless, the process of explaining detailed procedures and their complications is associated with certain problems. Occasionally, conveying complex medical information can be difficult and can adversely affect patients [9-11]. Specifically, it has been shown that patients were often more anxious when point by point information were revealed before surgical removal of impacted third molars [12]. The details for surgical removal of impacted mandibular third molars usually contain information about undesirable outcomes, such as temporary or permanent sensory nerve damage, dry socket, infection, haemorrhage, trismus, fracture of the mandible, iatrogenic damage to the adjacent second molar, and pain. Disclosing such information to patients before surgery could be stressful and elevate anxiety levels [13]. Thus, implementation of audio-visual aids to standardize the information about surgery as proposed in the literature is advised. It has been documented that audio-visual presentation prompts high patient fulfilment given the feasibility, convenience, and accessibility of data. There is no convincing evidence showing a decrease in preoperative anxiety. In contrast, it has been found that educating patients about the treatment with video can enhance patient knowledge about postoperative consequences and help in easing anxiety after the surgical removal of an impacted mandibular third molar [1].

The aim of the present study was to evaluate whether the conventional (verbal) method of explaining details of surgery or the audio-visual presentation could affect the anxiety level of patients who were scheduled for extraction of impacted mandibular third molars at our institute.

\section{Materials and Methods}

All patients who were referred to the Oral and Maxillofacial Clinic of the dental faculty (female Campus) at King Saud University, from 15 March 2017 to 30 June 2018, for the removal of impacted third

${ }^{\star}$ Correspondence to: Randa Alfotawi, Oral and Maxillofacial Department, Dental Faculty, King Saud University, Riyadh, Saudi Arabia, Tel: +96618056632; E-mail: ralfotawei@ksu.edu.sa

Key words: third molar, removal, anxiety, randomized trial, oral surgery

Received: January 31, 2019; Accepted: February 08, 2019; Published: February 12,2019 
molars were considered for inclusion in the study. Surgical removal of an impacted third molar was performed under local anaesthesia. A randomized clinical trial was planned; the study was independently reviewed and approved by the Dental Faculty Ethical Review Board (DERB) at King Saud University and complied with the rules related to the 'Research Ethics on Living Organisms' issued by Royal Decree no. M/29 and with the World Medical Association's Declaration of Helsinki. Patients, or their formal guardians in cases involving young adults (under 17 years of age), provided verbal consent; this protocol was advised and approved by the DERB. The consent form was prepared in accordance with the Research Ethics Review Committee of the World Health Organization.

A total of 40 patients scheduled to undergo extraction of impacted mandibular third molars were enrolled in this study. The inclusion criteria were the need for surgical extraction of third molar teeth, negative medical history, no current medication use, and age 20-45 years. The patients consented to participate in the study and were blinded to the study objectives. Each patient provided his or her sociodemographic information (gender, age, educational level, income, and Body Mass Index (BMI)). Four age groups were created including under 20 years, 20- 29 years, 30-39 years and above 40 years. Sample size calculation was follow well established protocol [14]. Patients were asked to indicate their-educational level as intermediate, secondary or college/university. With regards to income, the participants were asked to choose their approximate monthly income from four different groups as stated: less than SR 5000, SR 5000-SR 10000, SR10, 000- SR 15000 and above SR 20000. The weight and height for each patient were recorded to calculate their BMI, and patients were accordingly categorized as underweight, normal weight, overweight, or obese.

The patients were randomly designed at parallel group, with 1:1 allocation ratio based on their hospital file numbers. Files Number started with Even digit, they were subjected to verbal information about the stages of surgery and the anticipated postoperative complications (group1=20 participants) and file number started with odd digit, the patients received audio-visual information about the same topics (group2 $=20$ participants). These processes of patient allocation was carried out by cheif nurse who was blinded about the study objectives.

The narrated video shows simple illustrations created with Corel Video Studio Pro X9 by a graduated dental student at King Saud University. Audio and visual cues were included to describe the surgical procedure and postoperative complications, and a clear explanation was provided in non-technical language. Both the verbal and the audio-visual information were similar. All questions from the patients in both groups were answered by the surgeons. To minimise possible confounding effects, such as differences in background training, two surgeons treated all patients with standardized surgical procedures. The surgeons were trained in the content and proper delivery of the information to the patients.

\section{Modified Dental Anxiety Scale (MDAS)}

Patients were asked to rate their anxiety level based on a Modified Dental Anxiety Scale [15]. The scale was used only when the patient was seated on the dental chair and ready for surgery. This scale was chosen because it is reliable and considers anxiety during local anaesthesia injection rather than the Corah MDAS [16].

The patients were asked to score their anxiety levels through various situations on a scale from 0 (no anxiety at all) to 5 (maximum anxiety). The patients were asked to complete the scale at the following
8 different time points: immediately once the patient was seated on dental chair, after giving instructions, after injection, before extraction, during drilling, after extraction, during suturing, and before leaving the clinic. The data collection form used for all participants is shown-in Figure 1.

\section{Heart rate (HR) recording}

A HR sensor (POLAR H10, UK) detects beat-by-beat HR patterns, and a chest built recording strip was used (Figures 2 and 3). The sensor was placed directly on the skin of the subject (directly on the apex beat), and the POLAR H10 was found to be compatible with many Bluetooth devices. The sensor connects the HR to any application aiming to optimize the training or the workout of an individual. In our study, we connected it with and ActiGraph wGT3X-BT (USA) medical-grade wearable activity monitoring system, which has been deployed in many pharmaceutical drug trials around the world to capture high quality physical activity and sleep data. The activity monitors are FDA 510(k) approved Class II medical devices in the U.S. and adhere to regulatory standards worldwide. The other interesting feature of the POLAR H1O is its high precision due to improved electrodes that made Polar the most accurate heart rate sensor.

\section{Data collection table__HR research}

Part 1: demographics
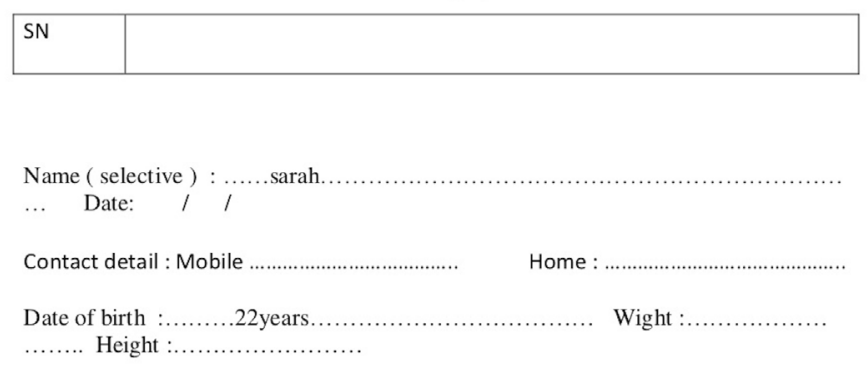

Education levels: $\square$ primary $\square$ intermediate $\square$ secondary $\square$ collage $\square$ above bhb

Maternal Status: $\quad \square$ married $\quad \square$ single $\quad \square$ Divorce $\quad \square$ Widow

Financial income : $\square$ less $5000 \quad \square$ 5000-10000 $\square$ 10000-15000 $\square$ above 20000

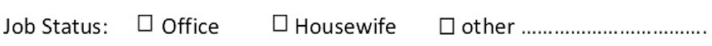

Part 2: Experiment

(verbal or video)

\begin{tabular}{|l|l|l|l|}
\hline Procedure & Time & Anxiety Scale & VAS \\
\hline $\begin{array}{l}\text { Immediately after } \\
\text { initialize }\end{array}$ & & & \\
\hline $\begin{array}{l}\text { After finishing } \\
\text { instruction }\end{array}$ & & & \\
\hline $\begin{array}{l}\text { After finishing } \\
\text { injection }\end{array}$ & & & \\
\hline $\begin{array}{l}\text { Before starting } \\
\text { extraction }\end{array}$ & & & \\
\hline $\begin{array}{l}\text { After finishing } \\
\text { extraction }\end{array}$ & & & \\
\hline $\begin{array}{l}\text { After end of } \\
\text { suture }\end{array}$ & & & \\
\hline Before leaving & & & \\
\hline
\end{tabular}

Figure 1. Shows the Clinical form that have used for data collection from each patien consented to enrol in the study, the table shows the variable surgical phases at which MDAS was taken 
Mean HR

\section{MEAN HR DURING SURGERY}

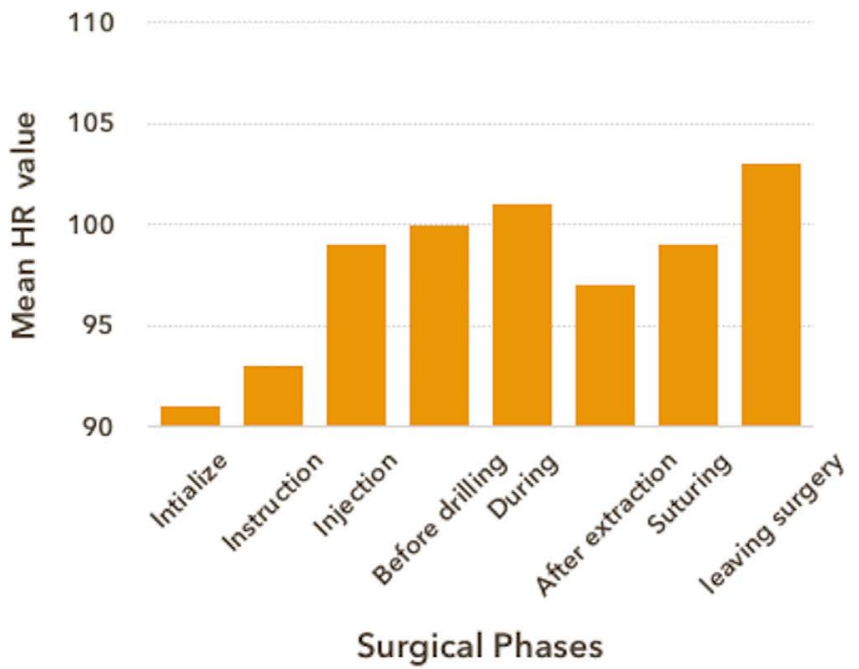

Figure 2. The devices that are used in the study. At right, POLAR H,. UK heart rate sensor and at left is the ActiGraph wGT3X-BT, USA activity monitor system. Both are Bluetooth connected

Mean HR

\section{MEAN HR DURING SURGERY}

$$
110
$$

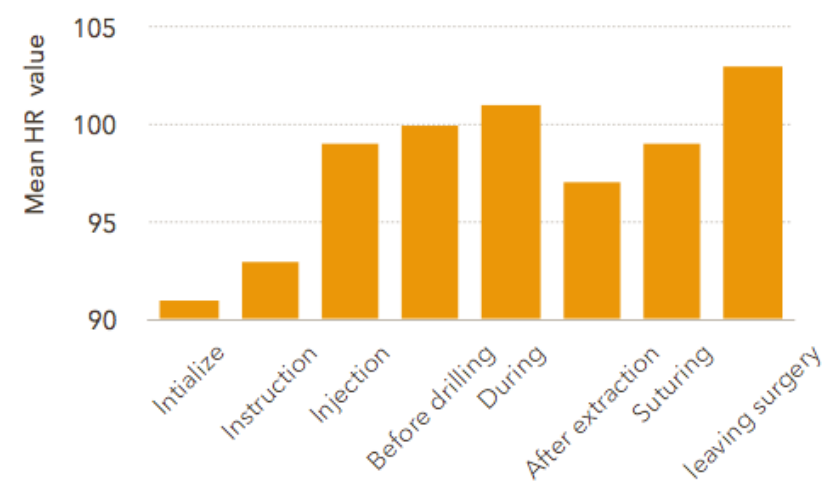

Figure 3. Graph shows the mean HR recording during different surgical phases for all patients

USB ANT sticks-wirelessly send recorded HR data to the computer during surgery. ActiGraph premiere data analysis software were used. ActiLife's robust screening and analysis toolkit allows-users to process and score collected data using a comprehensive selection of independently developed and validated algorithms. However, in this study, we limited the functionality of ActiLife's to HR monitors per seconds only.

\section{Data collection from ActiLife software}

A data table containing separate columns with corresponding headers for each data type collected by the device from each patient was generated. The author chose to use a single axis for Vector Magnitude.
A standard ${ }^{*}$.csv file produced during conversion simply exported the data into ${ }^{*}$.csv format. Data were exported as native ${ }^{*}$.AGD files to ${ }^{*}$.MAT format for easy importing into MathWorks MATLAB ${ }^{\star}$ mathematical software platform.

Another important point to consider was that the "Re-Integrate AGD File" option allows users to integrate AGD files to larger epoch periods (e.g., 1 second epoch data collection reintegrated to 60 second epoch periods). We chose to use 3 seconds, which means that the HR was integrated every 3 seconds. The data were then exported to any format directly from the AGD file viewer by clicking on "Export data". Data were exported to SPSS for statistical analysis.

\section{Statistics}

The results were analysed using parametric tests to determine whether there were statistically significant differences between the groups for the categorical variables. To compare the continuous variables (i.e., anxiety and physiological measures) between the groups across the 8 stages of data collection, we used mixed models for repeated measures (SPSS program) following an exploratory analysis and a selection of the best covariance structure. When the difference was significant, multiple comparison tests and Student's t-test were used to identify differences between the averages ( $p$-value $<0.05$ ).

\section{Results}

All participants met the above prescribed inclusion criteria and were randomly assigned either to watch the video presentation or listen to routine verbal explanation of procedures and the possible postoperative complications. Two patients refused to see the video prior to surgery as they claimed it would make them more anxious throughout the surgery. Our data showed a constant increase in participant HR during different phases of surgical procedures, namely, during injection, extraction, immediately after tooth removal, during suturing and upon leaving the clinic.

The highest reported mean for HR was $103.5 \pm 27 \mathrm{bpm}$ before leaving the clinic while the highest mean for MDAS was $3.4 \pm 1.4$ during drilling. The mean MDAS before drilling and immediately after extraction were $2.7 \pm 1.4$ and $2.7 \pm 1$, respectively (Figure 4 ).

When-comparing HR for the two groups (verbal( $\mathrm{N}=22)$ vs. video $(\mathrm{N}=18)$ ), it was found that those who watched-the video before surgery had lower $\mathrm{HR}$ reading compared with those who received verbal instructions. These differences in mean HR reading were statistically significant for the following surgical stages: during drilling, suturing, and upon leaving the clinic (Figure 5). On the contrary, MDAS was found to be inversely proportionate to HR records. Patients who were subjected to the video reported higher anxiety scores throughout all surgical stages compared with the other group. However, these differences were not statistically significant.

When we looked at the demographic variables, we found a relationship among the recorded HR, income, BMI, and level of education. We found a significant difference in MDAS score level in relation to different independent groups based on their income. The highest scores were reported for the income group above SR20,000 and SR10,000-15,000 at $4.3 \pm 0.3$ and $4.1 \pm 0.5$ during the drilling stage, respectively. On the other hand, the income group of less than 5000 per month showed the highest $\mathrm{HR}$ reading during suturing and upon leaving the clinic with $116 \pm 30$ and $122 \pm 24$ successively (Figure 6 ). Nonetheless, in both assessments there were no reported significance differences between the groups. 


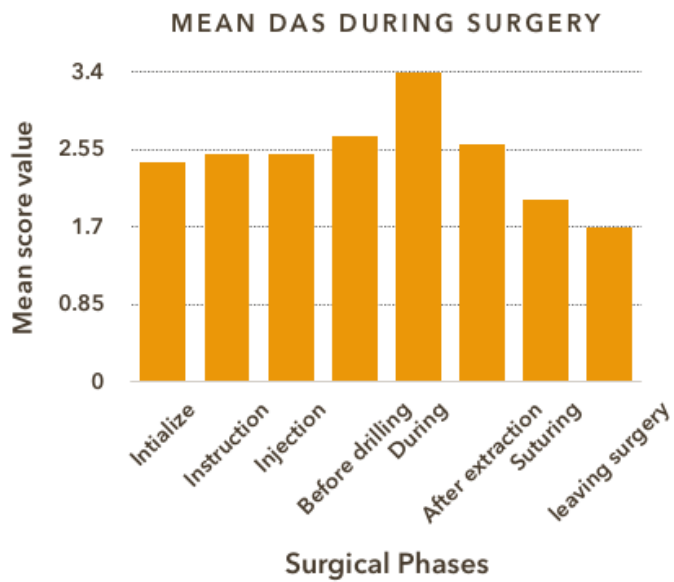

Figure 4. Graph showing the mean MDAS recorded for all patient at different surgical phase

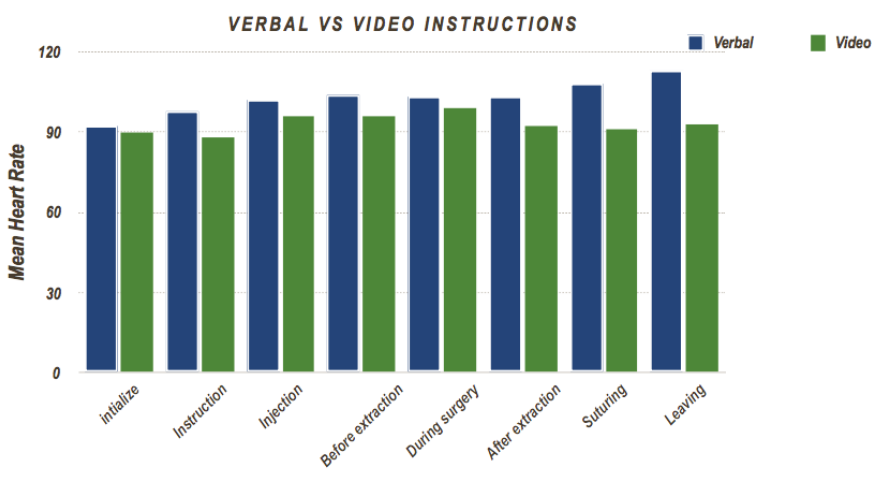

Figure 5. Graph showing the mean HR recorded for the two study groups who received either verbal or audio-visual instructions

When we looked at the demographic variables, we found a relationship among the recorded HR, income, BMI, and level of education. We found a significant difference in MDAS score level in relation to different independent groups based on their income. The highest scores were reported for the income group above SR20,000 and SR10,000-15,000 at $4.3 \pm 0.3$ and $4.1 \pm 0.5$ during the drilling stage, respectively. On the other hand, the income group of less than 5000 per month showed the highest HR reading during suturing and upon leaving the clinic with $116 \pm 30$ and $122 \pm 24$ successively (Figure 6). Nonetheless, in both assessments there were no reported significance differences between the groups.

With regards to the level of education, participants who had an intermediate education had higher initial HR recordings and at injection, before extraction and upon leaving when compared with participants who had college education. However, these differences were not statistically significant among the groups (Figure 7). In contrast, participants who finished their college education had higher MDAS at the following stages: initially, before extraction, during drilling, after extraction and during suturing. These data revealed statistically significant differences in mean scores between the groups.

In addition, the BMI variable was appraised. The obese group who had BMI values $>30$ had the highest HR recordings (Figure 8 ). The differences in means were statistically significant from injection until leaving the clinic at $\mathrm{p}<0.05$. Overweight and underweight patients had an MDAS score of $4 \pm 1.4$ at injection and drilling phases, and this value was statistically significantly different at $\mathrm{p}$ value $<0.05$.
Finally, the correlation between HR and MDAS was investigated. The data demonstrated a positive correlation within all surgical phases except at suturing at the 0.05 level (Figure 9).

\section{Discussion}

The present study sought to determine whether the use of an audiovisual presentation of surgical information would-improve-patient knowledge of postoperative complications and lessen anxiety during the removal of an impacted mandibular third molar in our speciality clinic at Dental faculty, KSU. Data were compared with a group who received verbal information as a typical daily practice in our surgical unit. The authors hypothesized that the audio-visual informed group would better understand the postoperative complications and exhibit less anxiety before and after surgery in comparison with the verbal group.

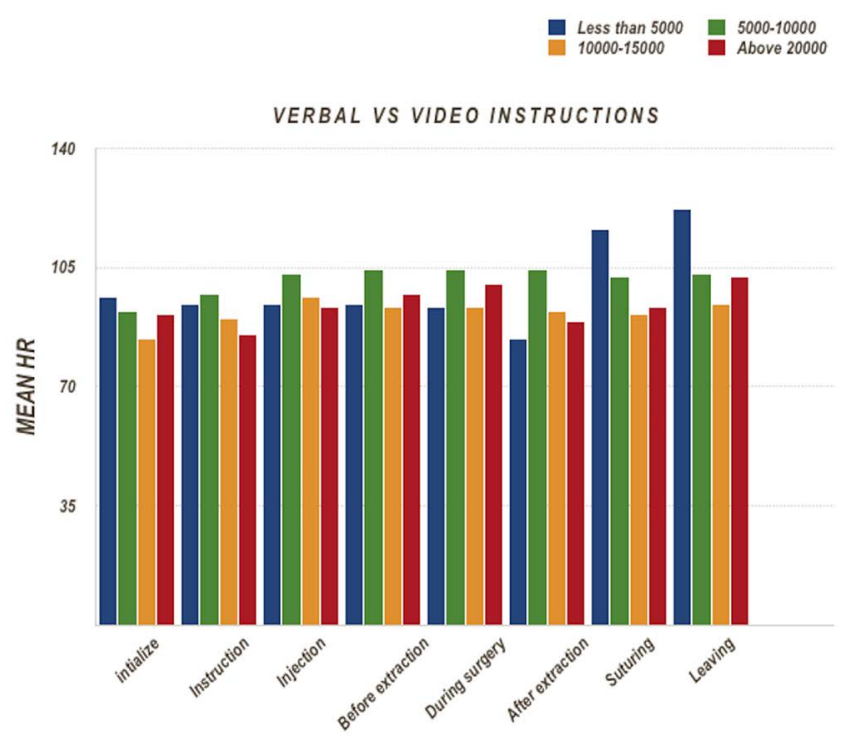

Figure 6. Graph shows mean HR for different groups based on their income at different surgical phases
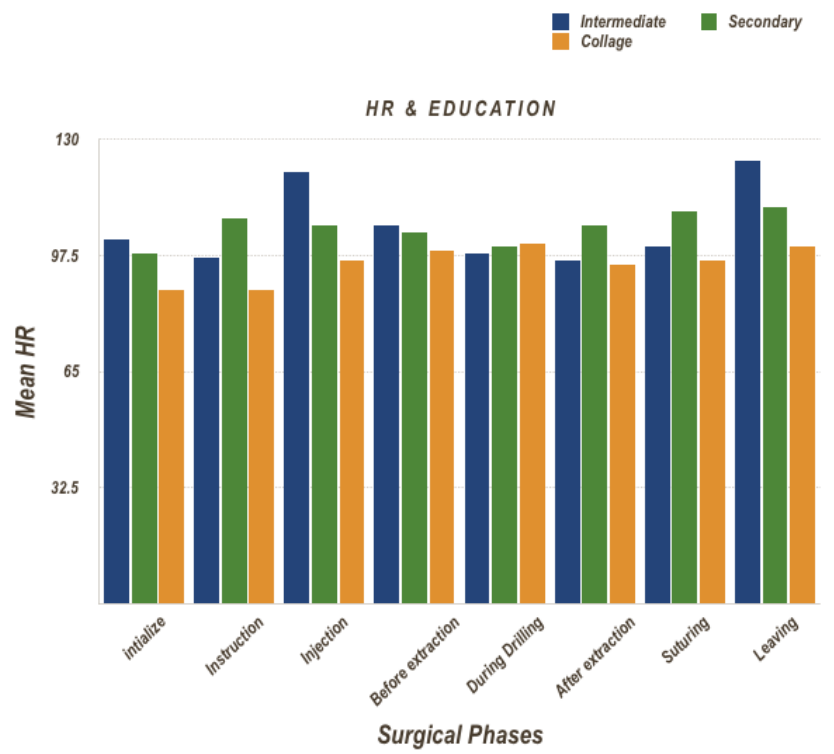

Figure 7. Graph showing the mean HR recorded based on patient level of education at different surgical phases 


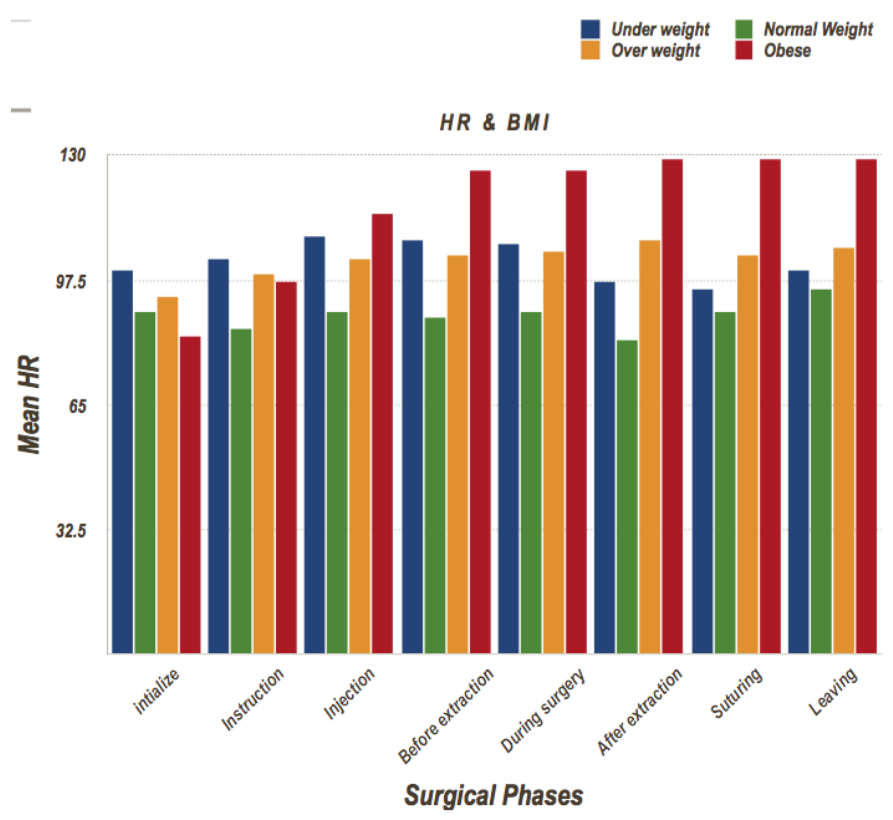

Figure 8. Graph showing the mean HR and BMI recorded for all patient at different surgical stages

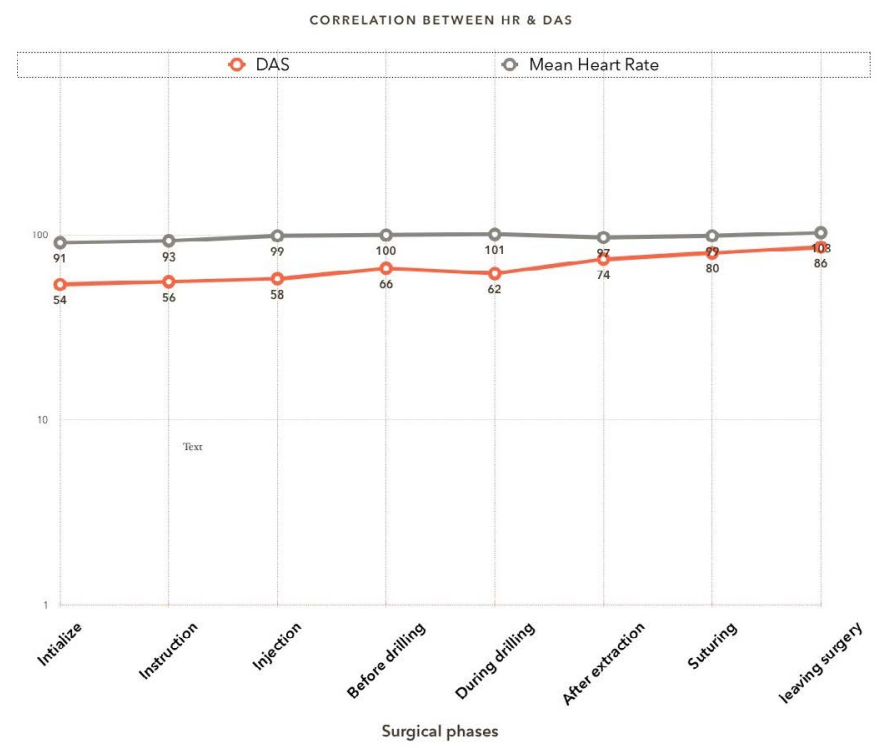

Figure 9. The linear relationship between the reported HR and the subjective MDAS score reported during different surgical phases

The novelty of this study is the recording of heart rate per second using POLAR H1 sensor-ActiGraph wGT3X-BT HR monitor and using the MDAS throughout the surgical phases. The MDAS was chosen because it is reliable, sensitive and easy to use in a clinical setting. The data showed lower HR readings for group who had been instructed with an audio-visual presentation compared with those who received verbal instructions. These differences in mean HR recording were statistically significant during most of the surgical phases. The data showed an increase in mean MDAS scores for those who were subjected to audiovisual slide information, with no statistically significant difference. A similar study design was conducted by Choi S, et al. [1], however they assessed DAS after one week from the day of surgery. Thus, they reported statistically significant differences between the groups.
These finding may infer that the audio-visual presentation viewed before surgery improved the understanding of potential postoperative complications. Additionality, a balanced amount of information was usually delivered in the video with a calming tone of voice, which are paramount factors as it has been found that overdetailed listing and disclosure before extraction of impacted mandibular third molars can exaggerate patient stress [11]. Heart and pulse rates have been used extensively in the literature as parameters for recording the autonomic system response to stress and level of anxiousness in other studies $[12,16,17]$. In this study, HR was monitored during different surgical stages and reported every 3 seconds. In addition, a reliable subjective tool to measure anxiety (MDAS) was considered.

Concerning the demographic data, level of education and BMI significantly affected patient anxiety during drilling. Increased mean HR was observed in patients who had an intermediate level of education. This could be due to difficulty in understanding the surgical procedure, possible complications, or too many details about surgery, which these patients might not understand and would therefore make them more anxious.

For BMI, obese candidates had the greatest HR recordings, which could be partly due to physiological adaptation of their body systems. It is a well-known fact that increased blood pressure and HR values are noted in obese people, presented by a decrease in parasympathetic activity and prevalence of sympathetic activity [18].

Obese individuals reported the highest resting heart rate of young adolescent males [19]. Interestingly, in our study, MDAS scores were higher for under and overweight candidates at several phases of the surgery. The data for underweight individuals were not consistent with the noted HR, which has a close association with changes in body mass index extent. Thus, in underweight and overweight subjects, the versatile adaptability of autonomic cardiac function was lessened [20,21].

It was interesting to note the importance of conveying optimum amount of surgical details, postoperative instructions and possible complications, which minimised patient anxiety and thereafter reduced postoperative pain. These facts have been mentioned in previous studies $[22,23]$. Vallerand, et al. reported that by providing good amount of information and to make sure that the patients understand about the possible outcomes, could considerably provide relief from pain and satisfaction with pain control. Thus, preventing the use of increased amount of analgesics [22].

On the other hand, studies have shown that preoperative information provided in audio-visual presentations fails to minimise patient anxiety before or after third molar extraction. Kazancioglu, et al. [24] claimed that watching a movie about third molar extractions led to an increase in anxiety and pain during the postoperative period [24]. Furthermore, Torres-Lagares, et al. [25]. reported that information given in a video format increased patient anxiety and did not provide advantages for dental treatment [25]. Audio-visual presentation showing detailed surgical steps and information can cause apprehension and fear among patients, which in turn counterbalance the more tolerable situation provided by better understanding or enhanced knowledge [24]. In the later study, the authors used DAS, the Spielberger State-Trait Anxiety Inventory (STAI), and pain analogue scales. However, the test was performed before surgery, after surgery, and one week after surgery for pain scoring. Nevertheless, in our study, MDAS was performed throughout the phases of the surgery, and we used an objective tool to record HR beat-by-beat to monitor the autonomic system response in the body. Moreover, a simplified 
animation clip presentation for all surgical steps was presented. Having said that, we considered many variables like levels of education, age, finical status and BMI. Based on our results, we believed that an audiovisual presentation provided a concise amount of information that is required to control patient anxiety throughout the procedure compared with routine verbal instructions that are commonly delivered in our daily practice. Our audio visual presentation was designed by using standardized illustrations, bullets, large font sizes, and nontechnical language to facilitate better comprehension. Unfortunately, we did not include a postoperative pain assessment strategy in this study, however, such an assessment should be considered in the future.

Controlling patient anxiety is a paramount pre-requisite to achieve optimum dental service and should be implemented in routine dental practice. Our previous work showed that $3.6 \%$ of patients who attended clinic for third molar removal developed a vasovagal attack as the main complication reported by the treating surgeons [26]. Moreover, it has been shown that surgeons require more operative time when patients were anxious [2].

It tends to be contended introducing audio-visual presentation in our practice would add more burden with regard to time and cost when compared to routine verbal informed consent [27].

Nevertheless, the burden of time and cost required for audio visual presentation is negligible to what would be achieved by better understanding of the surgical procedure. Having said that an extent of claims result from an absence of communication between the surgeon and patient as opposed to malpractice [28]. Therefore, implementing such a system would provide an optimum service to the patients and minimize such a risk.

Furthermore, it has been shown that anxiety is related to the perception and tolerance of pain. The clinician's performance may be hundred by patient anxiety in spite of delivering comprehensive and optimal treatment [26]. Patients are more happy and satisfied when they are provided with reasonable information before the surgical procedure, which helps them understand the situation better. Despite the fact that most patients do not recollect a great amount of the information provided to them during the process of informed consent [1].

There were short-comings in this study that should be considered when interpreting the data. First, the present study used a convenience sample, rather than a random population-based sample, and the sample was quite small. Previous related studies had much larger sample sizes $[2,27]$. A larger cohort of patients might provide sufficient statistical power to detect a correlation between HR and MDAS. Second, the gender of the current study included only women, and this was because of logistic reasons, as the study was conducted in the female campus in our institute. Gender might influence the factors analysed in this study, as young women frequently report feeling more anxious than men $[15,20,25]$. Finally, a postoperative pain assessment strategy was not planned for this study.

In conclusion, our experience at our institute with audiovisual presentations could improve patient knowledge about the surgical removal of impacted mandibular third molar. This would decrease dental anxiety amongst patients, thereby reducing potential complications. Additional studies are needed to evaluate the postoperative pain scoring. Larger patient samples including males are recommended to validate the results of the present study.

\section{Conflict of interest}

None.

\section{Acknowledgements}

We would like to acknowledge College of Dentistry Research Centre (CDRC) for their support, thanks to the charge nurses at the Oral and Maxillofacial Clinic in the GUC Ms Jennifer, as well as the students and our colleagues at the Dental Faculty, King Saud University, Riyadh, KSA. There was no financial support for this study.

\section{References}

1. Choi SH, Won JH, Cha JY, Hwang CJ (2015) Effect of audio-visual treatment information on relieving anxiety in patients undergoing impacted mandibular third molar removal. J Oral Maxillofac Surg 73: 2087-2092. [Crossref]

2. Filewich RJ, Jackson E, Shore H (1981) Effects of dental fear on efficiency of routine dental procedures. J Dent Res 60: 533-535.

3. Deanfield JE, Shea M, Kensett M, Horlock P, Wilson RA, et al. (1984) Silent myocardial ischaemia due to mental stress. Lancet 2: 1001-1005. [Crossref]

4. Yucel A, Gecici O, Emul M, Oyar O, Gulsoy UK, et al. (2005) Effect of informed consent for intravascular contrast material on the level of anxiety: How much information should be given? Acta Radiol 46: 701-707. [Crossref]

5. Kanegane K, Penha SS, Munhoz CD, Rocha SG (2009) Dental anxiety and salivary cortisol levels before urgent dental care. J Oral Sci 51:515-520. [Crossref]

6. Pole N1 (2007) The psychophysiology of posttraumatic stress disorder: a metaanalysis. Psychol Bull 133: 725-746. [Crossref]

7. Bloomfield DM, Zweibel S, Bigger JT Jr, Steinman RC (1998) R-R variability detects increases in vagal modulation with phenylephrine infusion. Am J Physiol 274: H1761-1766.

8. Hayano J, Sakakibara Y, Yamada A, Yamada M, Mukai S, et al. (1991) Accuracy of assessment of cardiac vagal tone by heart rate variability in normal subjects. $\mathrm{Am} \mathrm{J}$ Cardiol 67: 199-204. [Crossref]

9. Alexander M (1990) Informed consent, psychological stress and noncompliance. Humane Med 6: 113-119

10. Stead M, Eadie D, Gordon D, K Angus (2005) "Hello, hello—-It's English I speak!": A qualitative exploration of patients' understanding of the science of clinical trials. J Med Ethics 31: 664-669. [Crossref]

11. Foëx BA (2001) The problem of informed consent in emergency medicine research Emerg Med J 18: 198-204. [Crossref]

12. Casap N, Alterman M, Sharon G, Sammuni Y (2008) The Effect of Informed Consent on Stress Levels Associated with Extraction of Impacted Mandibular Third Molars. $J$ Oral Maxillofac Surg 66: 878-881. [Crossref]

13. Nehme J, El-Khani U, Chow A, Hakky S, Ahmed AR, et al. (2013) The use of multimedia consent programs for surgical procedures. A systematic Review. Surg Innov 20: 13-23. [Crossref]

14. Kadam P, Bhalerao S (2010) Sample size calculation. Int J Ayurveda Res 1: 55-57. [Crossref]

15. Humphris GM, Morrison T, Lindsay SJ (1995) The modified dental anxiety scale validation and united kingdom norem. Community Dent Health 12: 143-150. [Crossref]

16. Corah NL, Gale EN, Illig SJ (1978) Assessment of a dental anxiety scale. J Am Dent Assoc 97: 816-819. [Crossref]

17. Kim YK, Kim SM, Myoung H (2011) Musical intervention reduces patients' anxiety in surgical extraction of an impacted mandibular third molar. J Oral Maxillofac Surg 69: 1036-1045. [Crossref]

18. Alemany-Martínez A, Valmaseda-Castellón E, Berini-Aytés L, Gay-Escoda C (2008) Hemodynamic changes during the surgical removal of lower third molars. J Oral Maxillofac Surg 66: 453-461. [Crossref]

19. Rossi RC, Vanderlei LC, Goncalves AC, Vanderlei FM, Bernardo AF, et al. (2015) Impact of Obesity on autonomic modulation, heart rate and blood pressure in obese young people. Auton Neurosci 193:138-141. [Crossref]

20. Yar T (2010) Resting Heart Rate and its relationship with general and abdominal obesity in young male Saudi University students. Pak J Physiol 6: 6-17.

21. Triggiani AI, Valenzano A, Ciliberti MA, Moscatelli F, Villani S, et al. (2017) Heart rate variability is reduced in underweight and overweight healthy adult women. Clin Physiol Funct Imaging 37: 162-167. [Crossref]

22. Vallerand WP, Vallerand AH, Heft M (1994) The effects of postoperative preparatory information on the clinical course following third molar extraction. J Oral Maxillofac Surg 52: 1165-1170. [Crossref] 
23. Ng SK, Chau AW, Leung WK (2004) The effect of pre-operative information in relieving anxiety in oral surgery patients. Community Dent Oral Epidemiol 32: 227235. [Crossref]

24. Kazancioglu HO, Tek M, Ezirganli S, Demirtas N, et al. (2015) Does watching a video on third molar surgery increase patients' anxiety level? Oral Surg Oral Med Oral Pathol Oral Radiol 119: 272-277. [Crossref]

25. Torres-Lagares D, Heras-Meseguer M, Azcarate-Velazquez F, Hita-Iglesias P, Ruiz-de-León-Hernández G, et al. (2014) The effects of informed consent format on preoperative anxiety in patients undergoing inferior third molar surgery. Med Oral Patol Oral Cir Bucal 19: 270-273. [Crossref]
26. Al Fotawi RAM, Philip MR, Premnath SN (2017) Assessment of the referral system for surgical removal of third molars at the Dental Faculty, King Saud University. Int Dent J 67: 360-370. [Crossref]

27. Kang EY, Fields HW, Kiyak A, Beck FM, Firestone AR, et al. (2009) Informed consent recall and comprehension in orthodontics: Traditional vs improved rea ability and processability methods. Am J Orthod Dentofacial Orthop 136: e1-e13. [Crossref]

28. Garip H, Abali O, Göker K, Göktürk U, Garip Y (2004) Anxiety and extraction of third molars in Turkish patients. Br J Oral Maxillofac Surg 42: 551-554. [Crossref]

Copyright: @2019 Alfotawi R. This is an open-access article distributed under the terms of the Creative Commons Attribution License, which permits unrestricted use, distribution, and reproduction in any medium, provided the original author and source are credited. 